\title{
Gas purifiers for closed-loop gas systems
}

\section{G.Saviano $^{1}$}

Sapienza Universita`di Roma and Laboratori Nazionali di Frascati dell'INFN

E-mail: giovanna.saviano@uniromal.it

\section{C.Lupi, M.Ferrini, R.R. Aurilio}

Sapienza Universita 'di Roma

\begin{abstract}
Results on a study on the filtering system used at the CERN Closed Loop recirculation gas plant currently in test at CMS experiment at the LHC are reported. This study is focused on the regeneration processes of the metals - including both oxidation and reduction reactions of Copper, Zinc and Nichel - and on the thermodynamics of the regeneration reactions. This study confirms the filtering system efficiency towards the $\mathrm{O}_{2}$. The findings of this study allow us to propose an optimized use of the purification system.
\end{abstract}

XI workshop on Resistive Plate Chambers and Related Detectors (RPC2012)

INFN-Laboratori Nazionali di Frascati, Italy

February 5-10, 2012

Speaker 


\section{Introduction}

Resistive Plate Counters (RPC) are used in the muon detector of the Compact Muon Solenoid (CMS) experiment [1] at the CERN Large Hadron Collider. RPC use fluorine-based gas mixture whose main component is Freon. Because of the high cost of Freon and the very large gas volumes involved in the full system, the design of the CMS RPC gas system is based on a recirculation system called the Closed Loop (CL) .

The absence of gas contaminants is of paramount importance in all gas detectors, and especially in RPC due to the high reactivity of F-based gas mix used [2,3]. A gas gain monitoring system [4] has been designed, built and being commissioned by the CMS RPC group for the fast and accurate monitoring of the working point, based on monitoring of the avalanche charge and of the chamber efficiency. The gas gain monitoring system is based on three single-gap RPC subdetectors flushed with clean gas mixture, CL mixture after purifiers before crossing CMS $\mathrm{RPC}$, and CL mixture from the return lines. The gas gain monitoring will provide a warning in case of a working point change [5].

A gas quality monitoring system is being developed by the CMS RPC group and it will perform chemical analyses such as Gas Chromatography (GC), High Pressure Liquid Chromatography (HPLC), $\mathrm{pH}$ meters, etc. following the warning condition released.

No aspect of the chemistry of purifiers used in CL is fully understood, nor the extent or the nature of contaminants with the exception of fluoridric acid, whose presence is established. Tests $[6,7]$ at the Gamma Irradiation Facility (GIF) $[8,9]$ showed the presence of HF, which is detected by accumulation methods or by the use of HPLC, or ionic column-equipped GC. A systematic investigation has been proposed aimed to fully clarify the chemistry of purifiers used in the CL[10]. Purifiers and filters were studied at the ISR test area during chamber testing with cosmic rays $[6,7,9,10,11,12,13,14,15,16,17]$ This study is focused on characterization of metallic filters reactions during filtration and regeneration in the CL.

\section{Filters}

With the original selection criteria being the filtering of $\mathrm{H}_{2} \mathrm{O}$ and $\mathrm{O}_{2}$, tests at GIF showed GC peaks of unknown pollutants that disappeared after the following purifiers:

- purifier 1: consisting of a 24-litre cartridge filled with 5A-Type molecular sieve manufactured by ZEOCHEM

- purifier 2: consisting of two 24-litre cartridges, each filled with a combination of metal filters:

- $\mathrm{Cu}$-Zn filter type R12 manufactured by BASF

- $\mathrm{Cu}$ filter type R3-11G manufactured by BASF

- Purifier 3: consisting of Ni AlO3 filter type 6525 manufactured by LEUNA. This purifier has been removed at the time of preparation of this paper. 


\subsection{Metals}

Metals oxidize according to the following reactions:

$$
\begin{array}{lll}
2 \mathrm{Cu}(\mathrm{s})+1 / 2 \mathrm{O}_{2}=\mathrm{Cu}_{2} \mathrm{O} & \Delta \mathrm{Gf}-147.8 \mathrm{~kJ} / \mathrm{mol}, & \Delta \mathrm{Hf}=-170.6 \mathrm{~kJ} / \mathrm{mol} \\
\mathrm{Cu}(\mathrm{s})+1 / 2 \mathrm{O}_{2}=\mathrm{CuO}(\mathrm{s}) & \Delta \mathrm{Hf}=-156,7 \mathrm{~kJ} / \mathrm{mol} & \Delta \mathrm{Gf}=-129,7 \mathrm{~kJ} / \mathrm{mol} \\
\mathrm{Zn}(\mathrm{s})+1 / 2 \mathrm{O}_{2}=\mathrm{ZnO}(\mathrm{s}) & \Delta \mathrm{Gf}=-318,3 \mathrm{~kJ} / \mathrm{mol} & \Delta \mathrm{Hf}=-348,28 \mathrm{~kJ} / \mathrm{mol} \\
\mathrm{Ni}(\mathrm{s})+1 / 2 \mathrm{O} 2=\mathrm{NiO}(\mathrm{s}) & \Delta \mathrm{Gf}=-211,7 \mathrm{~kJ} / \mathrm{mol} & \Delta \mathrm{Hf}=-239,7 \mathrm{~kJ} / \mathrm{mol}
\end{array}
$$

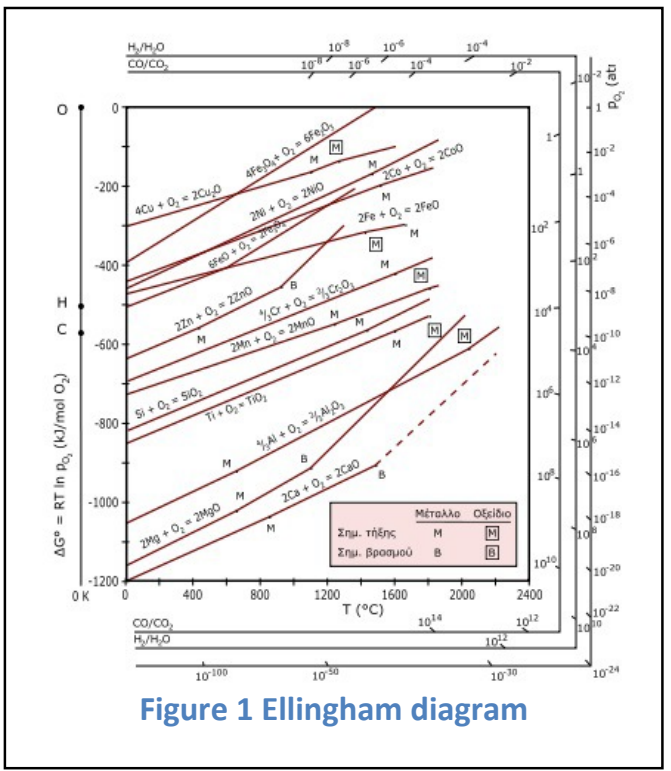

The Ellingham diagram (Fig.1) shows the high tendency of metals to oxidation. Thermodinamic parameters are calculated at $25^{\circ} \mathrm{C}$.

\subsection{Regeneration}

Metal filters are located as pellets inside the cartridges. Filters are provided as oxides, thus needing a regeneration before first use. The regeneration procedure consists of the following steps:

- A Noxal ${ }^{2}$ mixture flows through the cartridges, while it is gradually heated up to $150^{\circ} \mathrm{C}$;

- Further increase in temperature up to $215^{\circ} \mathrm{C}$

- Noxal flux stops; a fresh inert gas fluxes through the pellets until they reach room temperature.

\footnotetext{
${ }^{2}$ Gas mixture made of Argon and $\mathrm{H}_{2}(20 \%)$
} 
Since the materials' reduction has never been extensively studied in this system, a preliminary thermodynamic approach of the involved reactions was performed

$$
\begin{aligned}
& \mathrm{Cu}_{2} \mathrm{O}+\mathrm{H}_{2}(\mathrm{~g})=\mathrm{CuO}(\mathrm{s})+\mathrm{H}_{2} \mathrm{O}(\mathrm{g}) \\
& \mathrm{CuO}(\mathrm{s})+\mathrm{H}_{2}(\mathrm{~g})=\mathrm{Cu}(\mathrm{s})+\mathrm{H}_{2} \mathrm{O}(\mathrm{g}) \\
& \mathrm{NiO}(\mathrm{s})+\mathrm{H}_{2}(\mathrm{~g})=\mathrm{Ni}(\mathrm{S})+\mathrm{H}_{2} \mathrm{O}(\mathrm{g}) \\
& \mathrm{ZnO}(\mathrm{s})+\mathrm{H}_{2}(\mathrm{~g})=\mathrm{Zn}(\mathrm{s})+\mathrm{H}_{2} \mathrm{O}(\mathrm{g})
\end{aligned}
$$

\subsection{Thermodynamic study}

A first approach to the theoretical utility of the regeneration process used has been proposed starting from a thermodynamic study.

With the help of HSC Chemistry simulation software, in Fig. 2 is reported a graph of free energy versus temperature referred to the reactions between oxides and hydrogen, based on their data of enthalpy, entropy energy and equilibrium constant values of $\mathrm{CuO}, \mathrm{Cu}_{2} \mathrm{O}, \mathrm{ZnO}$ and $\mathrm{NiO}$. The temperature range in the simulation includes those used in the regeneration; the thermodynamic variables are calculated in steps of $20^{\circ} \mathrm{C}$.

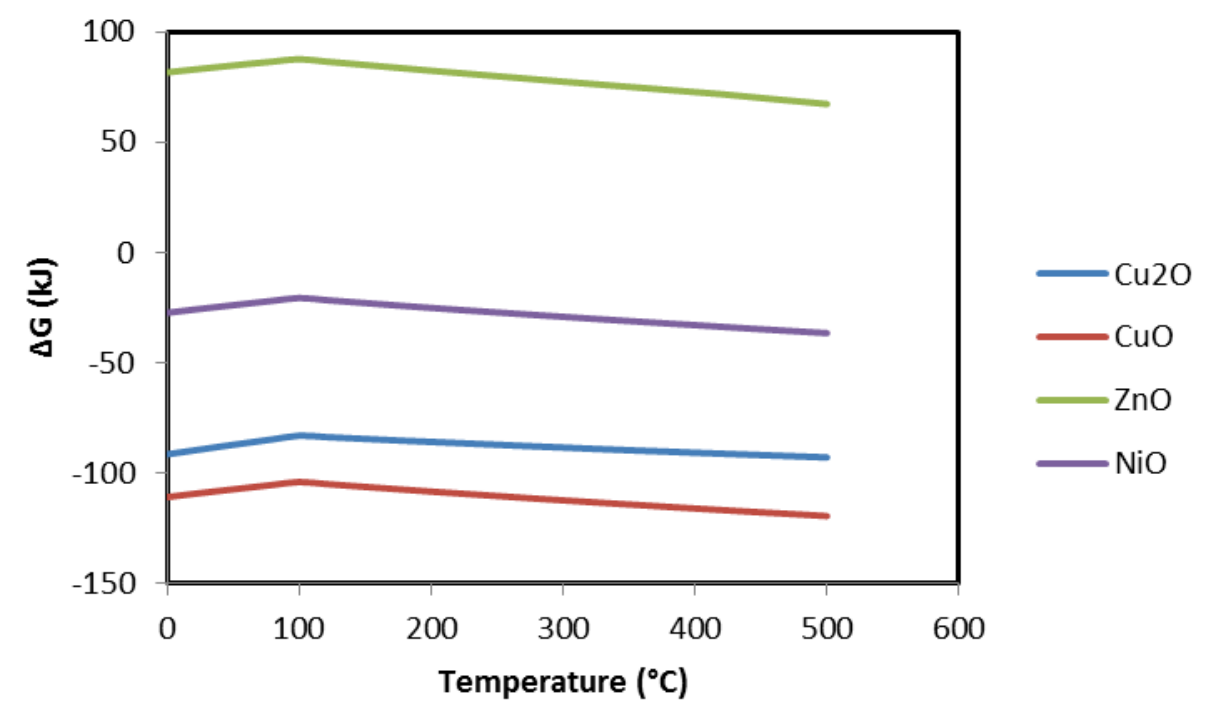

Figure 2 Free energies trend for all four reduction reactions. 
The most spontaneous reaction in the range considered is that of $\mathrm{CuO}$. As Figure 2 shows, the free energy trend is up to $100{ }^{\circ} \mathrm{C}$ increasing and then begin to decrease with the increasing in temperature. The different behavior is due to the presence of $\mathrm{H}_{2} \mathrm{O}$ vapor after $100{ }^{\circ} \mathrm{C}$ that implies a higher value of entropy.

\section{Conclusions}

The literature research performed [18-30], allowed us to formulate the following considerations

1. Filter $\mathrm{CuO} \mathrm{R} 311 \mathrm{G}$

Thermodynamic simulations highlight that $\mathrm{R} 311 \mathrm{G}$ filter mainly oxidizes to $\mathrm{Cu}_{2} \mathrm{O}$. From literature data we state the time needed to reach a 0.9 conversion of $\mathrm{CuO}$ :

- at $148^{\circ} \mathrm{C}$ the kinetic constant $\mathrm{k}_{0}=0.026 \mathrm{~min}^{-1}$ and the time needed is $95.6 \mathrm{~min}$;

- at $216^{\circ} \mathrm{C}$ the kinetic constant $\mathrm{k}_{0}=0.154 \mathrm{~min}^{-1}$ and the time needed is $16.1 \mathrm{~min}$. so the regeneration process can be optimized. Since the reduction time depends on the oxide mechanism formation, we can carry out the first regeneration in the worst condition. According to the data found in literature, $\mathrm{k}_{0}=0.0415 \mathrm{~min}^{-1}$ at $190^{\circ} \mathrm{C}$ is the smallest value of the kinetic constant. A 59.9 min time is necessary to reach a conversion of 0.9 in these conditions.

For later regenerations, we can assume that $\mathrm{CuO}$ is formed through a $\mathrm{Cu}_{2} \mathrm{O}$ oxidation; in such a case the regeneration can be carried out at $215^{\circ} \mathrm{C}$ for $20 \mathrm{~min}$ to have a conversion $>0.9$.

2. Filter $\mathrm{ZnO} \mathrm{R} 312$

It has the main aim to stop sulfur and arsenic eventually contained in the gas flow. These elements react with the filler in an irreversible way. When the amount of sulfur and arsenic in the gases will be known, we will be able to value the filler's life time.

3. Filter $\mathrm{Ni}-\mathrm{Al}_{2} \mathrm{O}_{3}$ Leuna 6525

Even the $\mathrm{NiO}$ regeneration process could be optimized. In fact at $215^{\circ} \mathrm{C}$, with the kinetic constant $\mathrm{k}_{0}=0.15 \mathrm{~min}^{-1}$, after 15.4 min we reach a conversion of 0.9 .

This purification step was redundant since it worked in the same way of the first step. It has already been removed from the CL of the RPC detector of the CMS experiment.

\section{References}

[1] CMS Collaboration (S. Chatrchyan et al The CMS experiment at the CERN LHC, JINST (2008) 3 (8), .

[2] J. Va'vra, "Physics and chemistry of aging: Early developments," ICFA Instrum. Bull. 24, 1 (2002) Nucl. Instrum. Meth. A 515, 1 (2003).

[3] J. Va'vra, "Summary of session 6: Aging effects in RPC detectors," Nucl. Instrum. Meth. A 515, 354 (2003).

[4] M. Abbrescia et al., "Gas analysis and monitoring systems for the RPC detector of CMS at LHC,” LNF-06-34-P, Jan 2007. 9pp. 2006 IEEE Nuclear Science Symposium (NSS/ MIC), San Diego, California, 29 Oct - 4 Nov 2006. arXiv:physics/0701014. 
[5] L.Benussi et al., The CMS RPC gas gain monitoring system: an overview and preliminary results, Nucl. Instrum. Meth. A 602 (2009) 805 [arXiv:0812.1108 [physics.ins-det]]

[6] M. Abbrescia et al., "HF Production In CMS Resistive Plate Chambers," Nucl. Phys. Proc. Suppl. 158 (2006) 30. NUPHZ,158,30;

[7] M. Abbrescia et al., "Study Of Long-Term Performance Of Cms Rpc Under Irradiation At The Cern Gif," Nucl. Instrum. Meth. A 533, 102 (2004).

[8] S. Agosteo et al., Nucl. Instrum. Meth. A 452 (2000) 94.

[9] M. Bosteels et al.,"CMS Gas System Proposal”, CMS Note 1999/018.

[10] M. Abbrescia et al., Proposal for a Systematic Study of the CERN Closed Loop Gas System Used by the RPC Muon Detectors in CMS, Frascati preprint LNF-06/27(IR), available at http://www.lnf.infn.it/sis/preprint/.

[11] L. Besset, F. Hahn, S. Haider, C. Zinoni, "Experimental Tests with a Standard Closed Loop Gas Circulation System", CMS Note 2004/004

[12] F. Hahn, Upgraded purifiers design for the full scale Closed Loop gas system of CMS RPC, CMS Note 2004/005

[13] LINDE Technical Bullettin.

[14] BASF Technical Bullettin

[15] LEUNA Data Sheet September 9, 2003, Catalyst KL6526-T.

[16] M. Abbrescia et al., HF production in CMS-Resistive Plate Chambers. 8th RPC2006, Seoul, Korea, 10-12 Oct 2005. Published in Nucl.Phys.Proc.Suppl.158:30-34,2006

[17] G. Aielli, P. Camarri, R. Cardarelli, A. Di Ciaccio, A. Di Simone, B. Liberti, L. Palummo, R. Santonico (INFN, Rome2 and Rome U.,Tor Vergata) Fluoride production in RPCs operated with F-compound gases.. 2006. 6pp. Prepared for 8thRPC2006, Seoul, Korea, 10-12 Oct 2005. In Nucl.Phys.Proc.Suppl.158:143-148,2006.

[18] Jose' A. Rodriguez, Jae Y. Kim, Jonathan C. Hanson, Manuel Pe'rez,and Anatoly I. Frenkel, Reduction of $\mathrm{CuO}$ in $\mathrm{H} 2$ : in situ time-resolved XRD studies. J. Am. Chem. Soc. 124 (2002) 346.

[19] Jae Y. Kim, Jose' A. Rodriguez, Jonathan C. Hanson, Anatoly I. Frenkel, and Peter L. Lee. Reduction of $\mathrm{CuO}$ and $\mathrm{Cu} 2 \mathrm{O}$ with $\mathrm{H} 2: \mathrm{H}$ Embedding and Kinetic Effects in the Formation of Suboxides Journal of the American Chemical Society. 09/2003; 125(35):10684-92. DOI:10.1021/ja0301673

[20] W. D. Bond W. E. Clark Technician: J. F. Talley, Reduction Of Cupric Oxide By Hydrogen. Fundamental Kinetics Oak Ridge National Laboratory Oak R i d g e, Tennessee ,Laboratory Records Department.M.D. McCluskey and S.J. Jokela Department of Physics, Washington State University, Pullman, WA 99164-2814.Hydrogen Donors In Zinc Oxide in Semiconductor

[21]Ashok, J. Chevallier, B.L.Sopori, M. Tabe, and P. Kiesel Defect Engineering-Materials, Synthetic Structures and Devices, edited by S. (Mater. Res. Soc. Symp. Proc. 864, Warrendale, PA , 2005), E10.4 
[22] Yixin HUA, F.R. Sale. Hydrogen Reduction and Microstructural Changes of ISF Sinter. Technol. 199612 (5): 389-394 ISSN: 1005-0302 CN: 21-1315/TG.

[23] S.Lew, A.F. Sarofim, M.Flytzani-Stephanopoulos. The Reduction of Zinc Titanate and Zinc Oxide Solids. Chemichal Engineering Science, Vol 47, nº, pp.1421 1431, 1992 Pergamon Press

[24] Fred A Weirich . A Study of the Reduction of Zinc Oxide by Hydrogen and Methane. http://scholarsmine.mst.edu/thesis/pdf/Weirich_09007dcc806d8b3d.pdf

[25] Jose' A. Rodriguez, Jonathan C. Hanson, Anatoly I. Frenkel, Jae Y. Kim, and Manuel Pe'rez. Experimental and Theoretical Studies on the Reaction of $\mathrm{H} 2$ with NiO: Role of O Vacancies and Mechanism for Oxide Reduction. Journal of the American Chemical Society. 01/2002; 124(2):346-54.

[26] R.P. Furstenau, G.McDougall and M.A.Langell. Initial Stages OF Hydrogen Reduction Of NiO(100). Surface Science, Volume 150, Issue 1, Pages L59-L121, 1-288 (1985)

[27] B. Jankovic, B. Adnaddevic, S. Mentus, The kinetic analysis of non-isothermal nickel oxide reduction in hydrogen atmosphere using the invariant kinetic parameters method. Thermochimica Acta, Volume 456, Issue 1, 1 May 2007, Pages 48-55.

[28] James T. Richardson, Robert Scates, Martyn V. Twigg. X-ray diffraction study of nickel oxide reduction by hydrogen. Applied Catalysis A: General DOI:10.1016/S0926$860 \mathrm{X}(02) 00669-5$ 